\title{
Thermodynamic properties of hydroxypropyl- $\beta$-cyclodextrin/guest interaction: a survey of recent studies
}

\author{
Federica D'Aria ${ }^{1} \cdot$ Bruno Pagano $^{1} \cdot$ Concetta Giancola ${ }^{1}$ (D)
}

Received: 14 March 2021 / Accepted: 14 June 2021 / Published online: 4 July 2021

(c) The Author(s) 2021

\begin{abstract}
For many years, cyclodextrins (CDs) have been the object of attention for their capability of improving the stability, solubility and bioavailability of numerous molecules of interest, including drugs and nutraceuticals. They have low toxicity and for this reason have been employed for different routes of administration, including oral, ocular, nasal and parenteral. Among them, the hydroxypropyl- $\beta$-cyclodextrin (HP- $\beta-\mathrm{CD}$ ) is the least toxic. Several physicochemical methodologies have been employed for studying cyclodextrin/guest interaction, but isothermal titration calorimetry (ITC) is the only one capable of simultaneously providing the binding constant, $\Delta H^{\circ}, \Delta S^{\circ}, \Delta G^{\circ}$ and the binding stoichiometry. Here, we present the state of the art of ITC studies applied to HP- $\beta$-CD/guest complexes, discussing selected publications of the last five years, highlighting the thermodynamic factors that are decisive for optimal encapsulation.
\end{abstract}

Keywords Hydroxypropyl- $\beta$-cyclodextrin · Isothermal titration calorimetry $\cdot$ Thermodynamics of interaction · Enthalpyentropy compensation

\section{Introduction}

Cyclodextrins (CDs) are largely studied for their application in enhancing stability, solubility and bioavailability of a variety of molecules, including drugs. CDs are natural products obtained by enzymatic degradation of starch and consist of cyclic oligosaccharides composed by $\alpha(1-4)$-linked D-glucopyranose of six, seven, or eight glucose units, named $\alpha, \beta$ and $\gamma$-cyclodextrins, respectively [1].

CDs have the shape of a truncated cone with a hydrophobic cavity and a hydrophilic surface with hydroxyl groups faced to aqueous solvents. They are able to include molecules of appropriate dimensions in the cavity, for application

This paper is dedicated to Guido Barone, our mentor, and Giuseppe Della Gatta, two eminent scientists in the field of calorimetry. They passed away, but their memory and passion for this matter live in us. Their teaching on how it is possible sharing research themes and friendship will remain in the minds and hearts of colleagues and alumni.

Concetta Giancola

giancola@unina.it

1 Department of Pharmacy, University of Naples Federico II, Via D. Montesano 49, 80131 Naples, Italy in the pharmaceutical, agricultural, food and nutraceutical industry.

The pivotal role of CDs in drug delivery has been object of several reviews [2-7]. A review on surfactant-cyclodextrin interaction, interesting for their application in many fields as self-assembly systems of amphiphiles, has also been recently published [8]. Here, we focussed our attention on 2 -hydroxypropyl- $\beta$-cyclodextrin (HP- $\beta$-CD) (Fig. 1 ), a synthetic hydroxyalkyl derivative, with better water solubility with respect $\alpha, \beta$ and $\gamma$-cyclodextrins, due to the presence of hydrophilic alcohol groups [9]. HP- $\beta-C D$ is the least toxic of the CDs, and it is well tolerated in humans, with no adverse events on kidney and only a mild problem of diarrhoea in the case of high dosage ( $>16 \mathrm{~g} /$ day) [10]. Usually utilized for oral administration, HP- $\beta$-CD has been approved by European Medicine Agency also for parenteral utilization (EMA 2017).

However, the efficacy of drug encapsulation in HP- $\beta-C D$ requires in-depth physicochemical studies on the binding interactions. Many factors, such as $\mathrm{pH}$, temperature and cosolvents, can affect the encapsulation due to the physicochemical properties of the guest molecule in the host-guest interaction.

This review is mainly focussed on the thermodynamic studies, based on isothermal calorimetry measurements, 
Fig. 1 Structure of 2-hydroxypropyl- $\beta$-cyclodextrin (on the left) and its schematic representation (on the right)
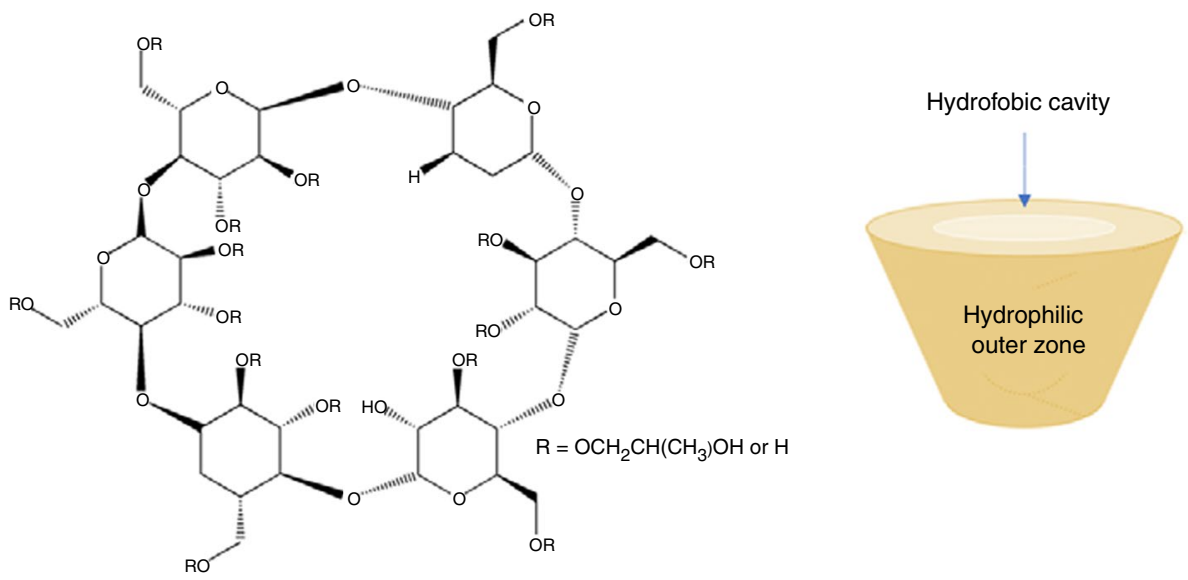

of hydroxypropyl- $\beta$-cyclodextrin complexation with guest molecules, appeared in the literature in the last five years. Recently, a book chapter has been dedicated to the thermodynamic properties of CD-drug interaction obtained by isothermal titration calorimetry [11]. That chapter was mainly focussed on the description of the calorimetric methodology supported by the example of HP- $\beta-C D$ complexed to the antihypertensive drug losartan. Here, we review recent articles in which the interaction of HP- $\beta$-CD with a different class of molecules has been studied by isothermal titration calorimetry, utilized alone or in combination with other methodologies.

Besides the paragraph dedicated to the common methodology, the articles have been discussed by grouping them into four paragraphs. All the studies reported here are certainly not rigidly framed in a single category, but some of them could be treated for their peculiarities in more than one paragraph.

\section{Calorimetric instrumentations and data analysis}

Isothermal titration calorimetry (ITC) is the main methodology to characterize any kind of biomolecular interaction, determining the heat changes occurring upon a binding process, experienced in our laboratory for different studies [12-16]. ITC measurements estimate with high precision the binding affinity $\left(K_{\mathrm{b}}\right)$, the binding stoichiometry $(\mathrm{N})$ and the binding enthalpy change $\left(\Delta H^{\circ}\right)$. The other parameters $\left(\Delta S^{\circ}\right.$ and $\Delta G^{\circ}$ ) can be easily calculated by the well-known thermodynamic relationships [17]. In the series of papers reviewed here, the most widely used instruments in the world were employed: VP-ITC and iTC200 microcalorimeters from Malvern Panalytical; Thermal Activity Monitor (TAM), TAM III, and Nano ITC (TA Instruments, USA). The experimental procedure is basically the same for all: one compound is titrated with the other one, both in solution at constant temperature (usually $298 \mathrm{~K}$ ). Whichever device is used, ITC is the most widely applied methodology to explore the molecular forces in the interaction between cyclodextrins and appropriate guest molecules to identify the best inclusion complex. In most of the papers described here, data analysis was made applying a single binding site model, since the stoichiometry was generally 1:1. Details on the relationships used for the data analysis can be found in each paper herein discussed.

\section{HP- $\beta-C D /$ drug interactions}

In a series of papers, the encapsulation of drugs in HP- $\beta-C D$ was studied for improving their solubility and bioavailability. In a work published in 2019, Hipólito-Nájera et al. studied the inclusion of indomethacin (IMC), a non-steroidal antiinflammatory drug, into both $\beta$-CD and HP- $\beta$-CD by ITC and $\mathrm{UV}-\mathrm{Vis}$ spectrophotometry, at two different $\mathrm{pH}$ values (pH 5 and 7) [18]. IMC has a very low solubility in water $\left(<0.01 \mathrm{mg} \mathrm{mL}^{-1}\right)$, and it was known that CDs improve its solubility. Although binding constants were obtained by different methodologies, a complete thermodynamic study had never been carried out before. Focussing on calorimetric measurements in phosphate buffer at $\mathrm{pH} 7$, the authors found that the association was entropically favoured for both CDs. The stoichiometry was $1: 1$, and binding constants were low: $\log K=2.81\left(K_{\mathrm{b}}=646 \mathrm{M}^{-1}\right)$ and $\log K=2.85$ $\left(K_{\mathrm{b}}=708 \mathrm{M}^{-1}\right)$, for $\beta-\mathrm{CD}$ and HP- $\beta-\mathrm{CD}$, respectively. The authors concluded that IMC has a slight preference for HP- $\beta-C D$, but although they report a very low error on log $K$ values, it is hard to assess that there are significant differences. However, their thermodynamic studies highlight the driving forces of interaction. One of these is certainly the displacing of water molecules from cavity for accommodating the IMC molecule, a process that increase the entropy.

Also in 2019, a paper describing the inclusion complex of $\beta-C D$ and HP- $\beta-C D$ with a series of antidiabetic nonsteroidal drugs was published [19]. The compounds are arylsulfonamido(benzo)thiazoles, and the most actives have a nitro group in position 4 of the sulphonamide moiety [20]. 
As regards the encapsulation on CDs, both the benzesulfonamide's phenyl group and benzothiazole can penetrate the hydrophobic cavity (Fig. 2).

ITC data show that the encapsulation is enthalpically unfavoured and entropically favoured, showing once again that the hydrophobic interaction is one of the main driving forces, which accounts for the positive signs of both the enthalpy and entropy change. The water displacing from hydration shells of hydrophobic interacting molecules is directly related to the positive entropy change. The entropy decreases on increasing the size of substituents as evidence of the fact that a minor number of water molecules is released from cavity. The binding constants are of the order of tens of units, and only one compound for each $\mathrm{CD}$ $(\beta-C D, H P-\beta-C D)$ is in the order of hundreds of units. When the compounds are capped with nitro and methoxy groups the affinity increases (higher binding constant). This finding was attributed to a sort of anchoring within the hydrophobic cavity. Overall, this paper well describes the host-guest interactions, but the advantage of using HP- $\beta$-CD rather than $\beta-\mathrm{CD}$ in delivering the studied compounds does not clearly emerge.

In two very recent papers, the thermodynamic studies of $\mathrm{HP}-\beta-\mathrm{CD} / \mathrm{drug}$ or $\beta-\mathrm{CD} / \mathrm{drug}$ interactions have been coupled to biological studies [21, 22]. The first one by Amaro et al. focuses on the synthetic antibiotic ofloxacin (OFLOX) [21]. In addition to ITC, the physicochemical methodologies utilized in this study were thermogravimetric and differential thermal analysis, Fourier transform infrared spectroscopy (FTIR) and proton nuclear magnetic resonance ( $\left.{ }^{1} \mathrm{H}-\mathrm{NMR}\right)$. Regarding the thermodynamic parameters, in line with the systems described so far, the obtained binding constants are low: $K_{\mathrm{b}}=65.2 \mathrm{M}^{-1}$ and $K_{\mathrm{b}}=880 \mathrm{M}^{-1}$ for OFLOX interaction with $\beta-C D$ and HP- $\beta-C D$, respectively. Enthalpy and entropy changes are both positive also for these complexes' formation. The interesting conclusions of these studies lie in the comparison with the microbiological and cytotoxic data, and the better biological result for HP- $\beta$-CD/OFLOX complex was correlated with its lower binding constant with a better drug release and consequently a better drug efficacy.

The second paper, by Muniz Cuoto et al., describes the combined effect of a two-drugs, a local anaesthetic (mepivacaine hydrochloride) and the analgesic capsaicin (CAP), on an inflamed tissue [22]. The idea comes from the finding that the local anaesthetic in acidic environment is charged and reduces its penetration in the cell. In this study, the ITC was used together with differential scanning calorimetry (DSC), X-ray, nuclear magnetic resonance (NMR), scanning electron microscopy (SEM) and phase solubility to describe the properties of HP- $\beta$-CD/CAP complex. Experiments in vitro and in vivo have been performed for evaluating the anaesthetic action of mepivacaine in the presence of HP- $\beta$-CD/CAP complex in acute inflammation. The study shows that this formulation works and could be a good alternative for clinical applications in local anaesthesia. What are the contributions of ITC and thermodynamic data to this study? The binding constant determined by ITC was 5100, quite higher than the systems discussed so far, and in disagreement with that obtained by phase solubility measurements. This discrepancy was attributed to the very different concentration ranges, under drug saturation conditions and in non-ideal solution for the phase solubility that could favour aggregation phenomena. Concerning the thermodynamic parameters, only the enthalpy change is mentioned $\left(\Delta H^{\circ}=-0.7 \mathrm{kcal} \mathrm{mol}^{-1}\right)$ and no comments on the other thermodynamic parameters are reported. We calculated $\Delta G^{\circ}$ and $\Delta S^{\circ}$ from the well-known thermodynamic relationships $\left(\Delta G^{\circ}=-\mathrm{RT} \ln K\right.$ and $\left.\Delta G^{\circ}=\Delta H^{\circ}-\mathrm{T} \Delta S^{\circ}\right)$ and, converting $\Delta H^{\circ}$ from calories into joules, we calculated $\Delta G^{\circ}=-21.2 \mathrm{~kJ} \mathrm{~mol}^{-1}$ and $\Delta S^{\circ}=71.1 \mathrm{JK}^{-1} \mathrm{~mol}^{-1}$. Summarizing, the encapsulation of capsaicin into HP- $\beta-C D$ is both enthalpically and entropically driven.

Very recently, the antibiotic doxycycline (DOX) has been encapsulated into HP- $\beta$-CD to enhance the activity against Aggregatibacter actinomycetemcomitans, a bacterium involved in periodontal diseases [23]. ITC has been employed for these studies, together with other methodologies (2D ${ }^{1} \mathrm{H}$ NMR, dynamic light scattering, zeta potential and SEM). Bacteria biofilm inhibition by DOX/HP- $\beta-C D$ complex has also been checked. In ITC measurements, DOX/HP- $\beta$-CD complexes, with different stoichiometry, were titrated in a suspension of Aggregatibacter actinomycetemcomitans. In this study, ITC measurements showed that the increase of the HP- $\beta-C D$ proportion in the complex induces changes in the binding profile, and the authors interpreted this result as a more efficient adhesion of cyclodextrin
Fig. 2 Benzothiazole (a) and benzesulfonamide (b) groups of arylsulfonamido(benzo)thiazole compounds

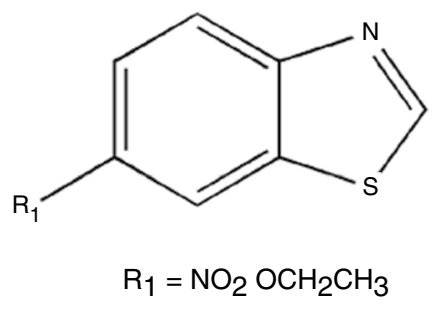

(a)

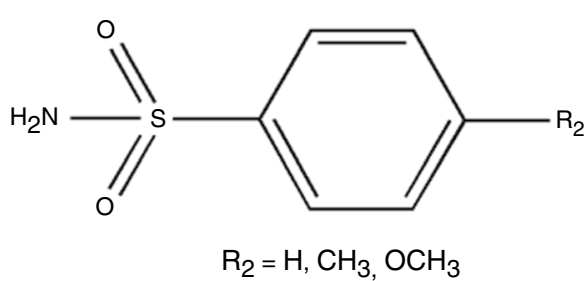

(b) 
with bacterium membrane. Hence, ITC has been utilized only for a qualitative information since the thermodynamic parameters have not been extracted.

In summary, the main advantage of HP- $\beta-C D$ in pharmaceutical application is to enhance the solubility of drugs and to increase their stabilities. ITC is very important because provides the complete thermodynamic picture of drug encapsulation. A spectroscopic description can be coupled to it, but above all a biological evaluation of the pharmacological properties is necessary. An important consideration derives from the works presented above: a relatively low binding constant is an advantage for effective drug release.

\section{HP- $\beta$-CD interaction with nutraceuticals/natural products}

In the last years, nutraceuticals have attracted the attention of researchers and many products based on these compounds; almost all molecules of natural origin have been put on the market and their business is quite prosperous. Nutraceuticals are compounds mainly used to improve health, increase life expectancy, and prevent diseases (for more information, see a recent review [24]).

The structures of the molecules interacting with hydroxypropyl- $\beta$-cyclodextrin described in this paragraph are shown in Fig. 3. In these studies, the stoichiometry of HP- $\beta$-CD/guest complex was 1:1 in all cases.

Phloridzin is a nutraceutical extract from apples and Lithocarpus polystachyus Rehd, a Chinese sweet tea. It possesses many properties as antioxidant, antidiabetic, antitumor and anti-inflammatory [25]. Its utilization in medicine, cosmetics and food is hindered by its low solubility; for this reason, the encapsulation of phloridzin into HP- $\beta-C D$ was studied by ITC in combination with spectroscopic methodologies (UV, IR and NMR) and molecular docking simulations [26]. ITC data were compared with those of $\beta-\mathrm{CD} /$ phloridzin complex. For both the CDs, the interaction was enthalpically driven, being negatives the $\Delta S^{\circ}$ values. The binding constants are relatively higher, $K_{\mathrm{b}}=1485 \mathrm{M}^{-1}$ and $K_{\mathrm{b}}=2019 \mathrm{M}^{-1}$ for $\beta-\mathrm{CD}$ and HP- $\beta-\mathrm{CD}$, respectively. The authors conclude that HP- $\beta-\mathrm{CD} /$ phloridzin formulation is promising for application in food industry and the thermodynamic parameters were attributed to the hydrophobic or van der Waals forces. In this case, it is hard to invoke the hydrophobic interactions since the $\Delta S^{\circ}$ is negative; rather, it is necessary to hypothesize as driving force the interactions of the hydrophilic type, and the formation of host/guest hydrogen bonds could be plausible to justify the negative $\Delta H^{\circ}$.

In a paper from our group, the inclusion of quercetin into HP- $\beta-C D$ was studied by isothermal calorimetry, DSC and phase solubility at $\mathrm{pH} 3.6$ and 8.0 [27]. Quercetin can be obtained by several fruits and vegetables and is able to induce anticancer activity. The binding constant $\left(K_{\mathrm{b}}=489 \mathrm{M}^{-1}\right)$ obtained by calorimetry at $\mathrm{pH} 8.0$ was in good agreement with that calculated by phase solubility. The interaction was both enthalpically and entropically driven, with the $T \Delta S^{\circ}\left(12.5 \mathrm{~kJ} \mathrm{~mol}^{-1}\right)$ predominant on $\Delta H^{\circ}$ $\left(-2.9 \mathrm{~kJ} \mathrm{~mol}^{-1}\right)$ value, confirming that the hydrophobic interactions are prevailing also for this complex formation.

The hydrophobic predominant interactions have been described also for the inclusion complex of HP- $\beta-C D$ and
Fig. 3 Schematic representation of inclusion complex between HP- $\beta-C D$ and a phloridzin, $\mathbf{b}$ oncocalyxone $\mathrm{A}, \mathbf{c}$ betulin, $\mathbf{d}$ rosmarinic acid, e caffeic acid, $\mathbf{f}(+)$-catechin and $\mathbf{e}(-)$-epicatechin (g)

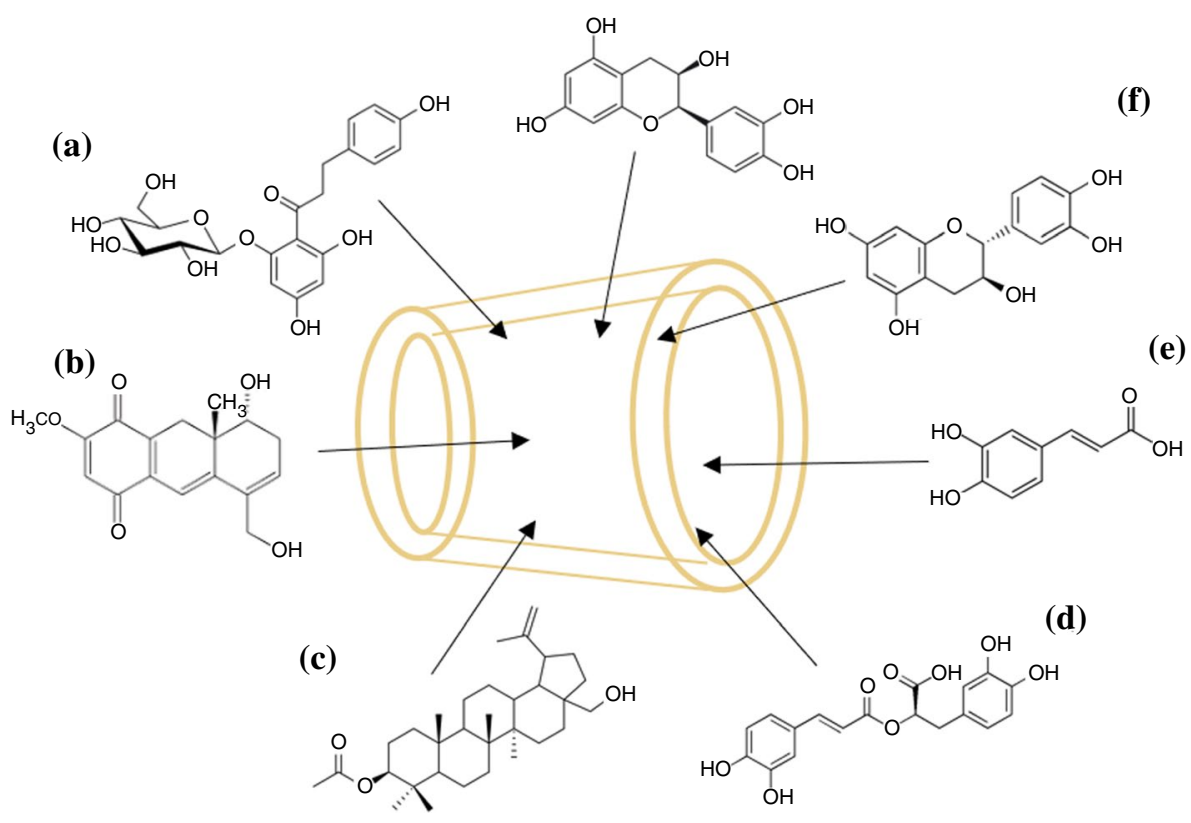


other CDs with oncocalyxone A (onco A) [28]. Onco A is a natural product, a quinone isolated from Auxemma oncocalyx tree of northeast Brazil and endowed with many biological activities [29]. As for the other compounds here reviewed, the bioavailability can be improved by complexation with CDs. In this study, onco A was checked for its encapsulation in different CDs, among these HP- $\beta-C D$, mainly by ITC with the complement of other techniques: FTIR, ${ }^{1} \mathrm{H}-\mathrm{NMR}$, DSC, TG and molecular modelling. However, the better interaction of onco A was found for HP- $\gamma$-CD based on binding constant values: $3175 \mathrm{M}^{-1}$ for HP- $\gamma$-CD, against $890 \mathrm{M}^{-1}$ for HP- $\beta-C D$. This preference was explained by docking simulation showing that the inclusion complex was stabilized by the formation of hydrogen bonds involving the oxygen atoms of onco $\mathrm{A}$.

HP- $\beta$-CD was also employed for increasing the solubility of betulin, a natural product extract from plants, mainly from the birch tree. HP- $\beta-C D /$ betulin complex was studied by calorimetry (DSC and ITC), IR spectroscopy and phase solubility. The hepatoprotective and anti-inflammatory activities have been also checked [30]. The complex formation, in line with many other HP- $\beta-\mathrm{CD} /$ guest systems, is both enthalpically and entropically favoured and the binding constant is $1330 \mathrm{M}^{-1}$. As for biological studies, the complex produces a liver protective effect in rats with non-alcoholic steatohepatitis at half dose with respect betulin alone.

Two natural food additives, caffeic and rosmarinic acids, known for their antimicrobial activity, have recently been studied for their inclusion in HP- $\beta$-CD [31]. As for all the other compounds here described, they are poorly soluble in water due to their hydrophobicity, and HP- $\beta-C D$ was utilized to improve solubility, bioavailability, and bioactivity. The two acids have a common caffeoyl moiety, but different physicochemical characteristics, which affects their interaction with HP- $\beta$-CD. The authors used different techniques for this study besides isothermal titration calorimetry, such as solid state and solution NMR, mass spectrometry and molecular dynamics. The thermodynamic data show interesting different behaviour. The binding constant of HP- $\beta$-CD/rosmarinic acid complex is two-fold higher than that of HP- $\beta-\mathrm{CD} /$ caffeic acid. This finding was corroborated by NMR and computational studies.

The complex formation is enthalpically driven for both acids, but $\Delta S^{\circ}$ is negative for caffeic acid and positive for rosmarinic acid, in agreement with a less polar character of the latter. These findings are well explained based on the release of structured hydration water to the bulk for explaining the positive entropy change, whereas for taking into account the negative enthalpy changes, the electrostatic interactions and $\mathrm{H}$-bonds were invoked as main driving factors.

In a couple of papers, HP- $\beta$-CD was checked for its capacity to differently capture phenethylamine enantiomers
[32] or two geometrical isomers: (+)-catechin and (-)-epicatechin [33]. In the first paper, the binding constants for a set of eight phenethylamine enantiomers and four cyclodextrins at $\mathrm{pH} 3.0$ were calculated by ITC and electrophoretic mobility data. The binding constants obtained with the two methodologies were in good agreement. The authors found that differences in enantiomer-CD complex mobilities are important for the separation of enantiomers, but the selectivity in the separation does not have a correlation with the values of binding constants.

On the other hand, Liu et al. showed that calorimetric measurements are able to discriminate between the two geometrical isomers: (+)-catechin (CA) and (-)-epicatechin (EC) [33]. These compounds are catechins contained in green tea and find application in foods and nutraceuticals as anti-inflammatory, anticancer, and in cosmetics [34, 35]. In this study, the thermodynamic data were combined with ${ }^{1} \mathrm{H}-\mathrm{NMR}$ and fluorescence data in the $\mathrm{pH}$ range of 6.8-8.0. The inclusion into HP- $\beta-C D$ of both compounds was enthalpically driven, being the $\Delta S^{\circ}$ values negative. The authors appropriately discussed the negative values of $\Delta S^{\circ}$ with the decrease of the rotational and translational degrees of freedom that overcomes the entropic gain due to the rearrangement of hydration water of both the host and the guest molecule. The main driving forces have been imputed to $\mathrm{H}$-bonds and van der Waals interactions. Finally, going back to the encapsulation difference between the two isomers, the author found that the binding constant of HP- $\beta-\mathrm{CD} / \mathrm{CA}$ complex is higher than that of HP- $\beta-\mathrm{CD} / \mathrm{EC}$ at the same temperature and $\mathrm{pH}$ value. Indeed, the comparison must be made in the same solution conditions, as $\underline{K}_{\mathrm{b}}$ value decreased with increasing temperature and $\mathrm{pH}$.

In conclusion, the increasing interest for nutraceuticals has led to many studies on the physicochemical properties of the encapsulation process of these molecules in cyclodextrins. The ITC studies have been often accompanied by other calorimetric studies (DSC), spectroscopic studies (NMR, IR, FTIR, etc.) and molecular docking. However, thermodynamic data serve as a guide to test the strength of encapsulation and the factors that govern it. Altogether, the various methodologies help to find the best conditions for an excellent formulation that pave the way for their commercialization.

\section{Improving the guest solubility in ethanol-water mixtures}

Many works discussed above use cosolvents, mainly dimethylsulfoxide and ethanol, to increase the solubility of HP- $\beta$-CD's guest and consequently to improve the encapsulation efficacy. Indeed, the use of addition of non-aqueous solvent to water is expected to help the solubilization of hydrophobic molecules by HP- $\beta$-CD. Particular care must 
be taken to perform calorimetric titration measurements in the presence of cosolvents. The exact same concentration of cosolvents must be used in the titrant and titrated, to avoid any spurious effects due to the presence of ethanol or dimethylsulfoxide, as large injection peaks due to dilution. Here, we discuss the papers published in these last years that systematically study the effect of ethanol-water mixtures. Starting from HP- $\beta$-CD/quercetin complex, previously studied in water [27] and recently studied in hydroalcoholic solution, we shortly discuss the calorimetric titration for the complex formation on changing the ethanol volume fractions at different $\mathrm{pH}$ values [36]. A schematic representation of HP- $\beta-C D / q u e r c e t i n$ inclusion complex is shown in Fig. 4.

Calorimetric titration showed that at ethanol molar fraction higher than 0.2 at neutral and alkaline $\mathrm{pH}$, and higher than 0.1 at acidic $\mathrm{pH}$, the complex does not form. These results indicate that ethanol competes with water for the cavity of HP- $\beta$-CD. DSC data of dried HP- $\beta$-CD, coming from hydroalcoholic solutions, confirm this hypothesis. In summary, the solubility enhancement of quercetin in higher concentrations of ethanol is counterbalanced by a lower propensity for the complex formation. A similar behaviour was found for the complex between HP- $\beta-C D$ and the natural product (E)-piceatannol (PIC), utilized in pharmaceutical and food industries [37]. Indeed, the binding constant decreases on increasing the alcohol concentration. In that study, methanol, ethanol, ethylene glycol, n-propanol, 1,3-propanediol and glycerol were utilized as cosolvents. Interestingly, the binding constant decreases on increasing the alkyl chain length but increases with the increasing the number of hydroxyl groups. For all systems studied, both enthalpy and entropy changes are negative; consequently, the complexation is enthalpically favoured. The authors attributed the negative entropy change to the predominant contribution of the loss of translational and rotational degrees of freedom of PIC in the cavity that exceeds the contribution of the water rearrangement/displacement upon complexation. The negative enthalpy change was ascribed to hydrogen bonds and van der Waals interactions between PIC and HP- $\beta$-CD. The displacement of water from the cavity was also mentioned to explain a negative $\Delta H^{\circ}$. This is a controversial question, which is better discussed in "Concluding

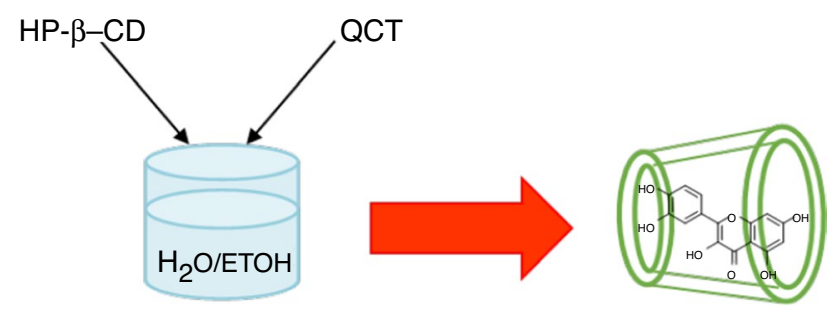

Fig. 4 Inclusion complex between HP- $\beta-C D$ and quercetin in ethanol-water mixtures remarks" paragraph. In addition, the presence of alcohol as cosolvent partially change the solvation cage around the interacting molecules and the increasing alcohol concentration renders less efficacious the desolvation effect and consequently decrease the complexation constant.

In a recent paper, the complex between curcumin and HP- $\beta$-CD was studied in water/ethanol solution by DSC, isothermal calorimetry, and phase solubility experiments at pH 5.0 and 7.4 [38]. Curcumin is a polyphenol endowed with many pharmacological properties, largely utilized in in traditional Chinese medicine, in foods and cosmetics but, as for the most part of molecules described along the text, possesses low water solubility. Therefore, a solution with $20 \%$ in volume of ethanol in water was utilized to improve the solubility at the two different $\mathrm{pH}$ values. Binding constant was slightly higher in acidic solution with respect to physiological solution and the complex formation was enthalpically and entropically favoured at both $\mathrm{pHs}$. The main result of this work is that in hydroalcoholic solution curcumin is successfully encapsulated in the cavity of HP- $\beta-C D$, with a preference at $\mathrm{pH}<\mathrm{pK}_{\mathrm{a}}$, whereas in water the complex does not form.

In summary, the combination of hydroalcoholic solution and encapsulation in HP- $\beta-C D$ is an effective strategy for improving the solubility of natural products endowed of health benefits. At the same time, the choice of an adequate $\mathrm{pH}$ and alcohol concentration is extremely crucial for the formation of the complex, as a competition for the HP- $\beta-\mathrm{CD}$ cavity among water, ethanol and the guest molecule is established above a certain percentage of alcohol. The thermodynamic data of host/guest interaction are certainly fundamental in finding the appropriate cosolvent quantity for an optimization of the complex formation in view of a pharmaceutical formulation.

\section{Enthalpy-entropy compensation}

In a couple of papers, the thermodynamics of $\mathrm{CD}$ complexes is deeply analysed highlighting the enthalpy-entropy compensation (ECC) [39, 40]. A premise is necessary to explain the ECC phenomenon. We can start from the obvious consideration that for a spontaneous process (in our case the host/guest interaction) the Gibbs energy must be negative. Structural modifications in interacting molecules show contributions of $\Delta H^{\circ}$ and $\Delta S^{\circ}$ to Gibbs energy which often work in opposition, i.e. if the enthalpy change is favourable the entropy change is unfavourable or vice versa, so that $\Delta G^{\circ}$ remains quite constant. The explanation for ECC lies in the participation of the solvation water molecules and their reorganization after interaction [41, 42].

In the paper of Paul et al. [39], the EEC has been discussed for the interaction between two bile salts and three $\beta$-CDs. Specifically, sodium deoxycholate $(\mathrm{NaDC})$ and 
sodium glycocholate (NaGC) were studied by ITC for their interaction with $\beta-\mathrm{CD}$, HP- $\beta-\mathrm{CD}$, and methyl- $\beta-\mathrm{CD}$. Heat capacity changes were also determined together with the binding constant and all the thermodynamic parameters. The binding constants are in the order of $10^{3} \mathrm{M}^{-1}$, and the complexation is both enthalpically and entropically favoured for all the complexes. The authors state that "the overall process is predominantly enthalpy-driven with small entropic contribution to the total free energy change", but this is only partially true because in almost half of the complexes the entropic contribution is greater than enthalpic one (in modulus). However, they discuss the favourable negative $\Delta H^{\circ}$ as a driving force for the inclusion of bile salts in HP- $\beta$-CD invoking the "non-classical hydrophobic effect" due to the release of high-energy water from cavity to the bulk (see the following paragraph for a more in-depth discussion). The binding was followed at different temperatures, and $\Delta G^{\circ}$ values were found quite constants on changing temperature for all the studied host/guest complexes. The authors discuss these results in terms of enthalpy-entropy compensation with temperature.

The observation of the ECC phenomenon has been also described in the paper of Schönbeck and Holm [40]. The inclusion of several water-soluble adamantane derivatives (ADs) into $\beta$-CD or HP- $\beta$-CD from different companies has been studied by NMR, ITC and molecular dynamics. Neutral and charged ADs were chosen for this study. Particularly interesting in this work is the choice of small guest molecules able in principle to be completely included in the cavity without protrusion from the cavity. This should permit to exclude the interactions of the host/guest surfaces to better study the factors that govern the efficacy of the inclusion in the cavity and verify the hypothesis that ECC phenomenon observed for host/guest complex is mainly imputable to dehydration of guest molecule. Nevertheless, they observed that going from $\beta$-CD to HP- $\beta-\mathrm{CD}, \Delta H^{\circ}$ value increases of $11-15 \mathrm{~kJ} \mathrm{~mol}^{-1}$ and $\mathrm{T} \Delta S^{\circ}$ of $6-10 \mathrm{~kJ} \mathrm{~mol}^{-1}$, depending on the guest. These differences show that ADs protrude from the $\mathrm{CD}$ cavity and interact with the sidechains at the rims of modified $\beta C D$. The authors conclude that the ADs are not so good model, as they hypothesized. However, their paper is of interest for the comprehensive discussion on enthalpy-entropy compensation.

In summary, direct interactions between the interacting molecules lead to a negative enthalpy change, but it is compensated by decrease in entropy due to the loss of conformational degrees of freedom. Chemical modifications in interacting systems involve changes in enthalpy compensated by change in entropy, so that the Gibbs energy change remains almost constant. This is the classic explanation for enthalpy-entropy compensation (EEC) phenomenon. However, changes in solvation are pivotal for understanding the ECC phenomenon [43], since they can also contribute to it.
The usual way to represent the ECC is to plot $\Delta H^{\circ}$ versus $\mathrm{T} \Delta S^{\circ}$ (or $\mathrm{T} \Delta S^{\circ}$ versus $\Delta H^{\circ}$ ), and the slope is expected to be equal to one for perfect compensation. In one of the papers discussed above [40], this plot for a series of adamantane derivatives interacting with HP- $\beta-\mathrm{CD}$ is reported and commented, but the slope for some derivatives is lower than unity, indicating a low degree of compensation. In the other work, the ECC is described in a plot of $\Delta H^{\circ}, \mathrm{T} \Delta S^{\circ}$ and $\Delta G^{\circ}$ versus temperature, which is a less usual way of representing it [39]. In any case, here it has been established that the EEC is a phenomenon not restricted to the protein/drug or nucleic acid/drug interaction, but that it is a general phenomenon that also affects guest/ HP- $\beta-C D$ interactions.

\section{Concluding remarks}

Isothermal titration calorimetry is a powerful methodology able to give all the thermodynamic parameters related to interacting (macro)molecules or systems in solution. It does not require reporter labels such as fluorophores and gives the possibility to perform experiments with not optically transparent solutions as dispersions or cells. On the other hand, it is necessity to take some precautions: the molecules in the syringe and in the cell must be in the exact same buffer to avoid large injection peaks due to dilution; if protonation/deprotonation is expected to be part of the reaction, a buffer with a low enthalpy change of protonation should be used; no other reactions should occur on the timescale of the experiment.

ITC is particularly precious to investigate host/guest complex formation, giving the complete thermodynamic picture in a single measurement. This information is very important to establish the ability of HP- $\beta-C D$ to encapsulate molecules of interest in many fields (foods, nutraceuticals, drugs, etc.). These molecules are generally scarcely soluble in water due to their chemical nature, so the experiments should be accurately designed with low concentration of guests. In some cases, to improve the solubility, experiments in mixed solvents could be designed, being careful to add only small quantities of cosolvents. Commonly used cosolvents include ethanol and dimethylsulfoxide, but the former can compete with the guest for encapsulation into cyclodextrin cavity at concentrations higher than $20-30 \%$ and the latter gives rise to large dilution peaks at concentrations higher than $5 \%$.

Generally, most of the HP- $\beta-C D /$ guest interactions are enthalpy-driven, while entropy may or may not be favourable. The stoichiometry of complexation is normally $1: 1$, and the binding constants are generally low, in the range $10-4000 \mathrm{M}^{-1}$. This is an advantage as it facilitates the release of the guest molecule. The inclusion process into HP- $\beta$-CD depends not only on the physicochemical properties of the guest, but also on the solution conditions (solvent, 
$\mathrm{pH}$, etc.) and the temperature. In any condition, it is crucial to focus on each host/guest system to identify the driving force of interaction. Commonly, the enthalpy change is attributed to hydrogen bonds, electrostatic forces and van der Waals interactions, whereas the entropy change is ascribed to reduction of conformational freedom and hydrophobic interactions.

Focussing on hydrophobic interaction, it is generally described with a release of ordered water from the hydration shell of interacting hydrophobic molecules with the loss of hydrogen bonds that leads to an unfavourable enthalpy change $\left(\Delta H^{\circ}>0\right)$ and to a favourable entropy change $\left(\Delta S^{\circ}>0\right)$ [44]. However, in the case of the inclusion of hydrophobic molecules in the hydrophobic cavity of HP- $\beta-C D$, a favourable enthalpy change, found in a paper described above, has been attributed also to a "non-classical hydrophobic effect" [39]. In few words, the displacement of water molecules from cyclodextrin cavity to accommodate the guest should result in a negative enthalpy change. This hypothesis is corroborated by a recent molecular dynamics simulation study [45] in which the authors found that the water molecules in the cavity form a smaller number of hydrogen bonds than bulk water, so that there is an enthalpy gain in the release of water from the cavity. However, we must not forget that a negative $\Delta H^{\circ}$ is the result of many concomitant interactions and that many molecules protrude from the cavity and can establish contacts with the rim of CDs, without invoking the phenomenon of "non-classical hydrophobic effect", which still remains controversial. In conclusion, the knowledge of the thermodynamic aspects of guest/CD complex formation in different solution conditions is essential to choose the best formulation in manifold fields of application and isothermal titration calorimetry is a hardly replaceable methodology for this purpose.

Funding Open access funding provided by Università degli Studi di Napoli Federico II within the CRUI-CARE Agreement.

Open Access This article is licensed under a Creative Commons Attribution 4.0 International License, which permits use, sharing, adaptation, distribution and reproduction in any medium or format, as long as you give appropriate credit to the original author(s) and the source, provide a link to the Creative Commons licence, and indicate if changes were made. The images or other third party material in this article are included in the article's Creative Commons licence, unless indicated otherwise in a credit line to the material. If material is not included in the article's Creative Commons licence and your intended use is not permitted by statutory regulation or exceeds the permitted use, you will need to obtain permission directly from the copyright holder. To view a copy of this licence, visit http://creativecommons.org/licenses/by/4.0/.

\section{References}

1. Tonkova A. Bacterial cyclodextrin glucanotransferase. Enzyme Microb Technol. 1998;22:678-86.

2. Davis ME, Brewster ME. Cyclodextrin-based pharmaceutics: past, present and future. Nat Rev Drug Discov. 2004;3:1023-35.

3. Loftsson T, Duchene D. Cyclodextrins and their pharmaceutical applications. Int J Pharm. 2007;329:1-11.

4. Otero-Espinar FJ, Torres-Labandeira JJ, Alvarez-Lorenzo C, Blanco-Méndez J. Cyclodextrins in drug delivery systems. J Drug Deliv Sci Technol. 2010;20:289-301.

5. Kurkov SV, Loftsson T. Cyclodextrins. Int J Pharm. 2013;453:167-80.

6. Jansook P, Ogawa N, Loftsson T. Cyclodextrins: structure, physicochemical properties and pharmaceutical applications. Int $\mathbf{J}$ Pharm. 2018;535:272-84.

7. Muankaew C, Loftsson T. Cyclodextrin-based formulations: a non-invasive platform for targeted drug delivery. Basic Clin Pharmacol Toxicol. 2018;122:46-55.

8. dos Santos Silva Araújo L, Lazzara G, Chiappisi L. Cyclodextrin/surfactant inclusion complexes: An integrated view of their thermodynamic and structural properties. Adv Colloid Interface Sci. 2021;289:102375.

9. Jambhekar SS, Breen P. Cyclodextrins in pharmaceutical formulations I: structure and physicochemical properties, formation of complexes, and types of complex. Drug Discov Today. 2016;21:356-62.

10. Gould S, Scott RC. 2-Hydroxypropyl- $\beta$-cyclodextrin (HP- $\beta-C D)$ : A toxicology review. Food Chem Toxicol. 2005; 43:1451-9.

11. Chatziathanasiadou MV, Mavromoustakos T, Tzakos AG. Unveiling the thermodynamic aspects of drug-cyclodextrin interactions through isothermal titration calorimetry. Methods Mol Biol. 2021;2207:187-98.

12. Pagano B, Mattia CA, Giancola C. Applications of isothermal titration calorimetry in biophysical studies of G-quadruplexes. Int J Mol Sci. 2009; 10:2935-57.

13. Giancola C, Pagano B. Energetics of ligand binding to G-quadruplexes. Top Curr Chem. 2013;330:211-42.

14. Fotticchia I, Fotticchia T, Mattia CA, Giancola C. Chitosan-based nanoparticles studied by isothermal titration calorimetry. J Therm Anal Calorim. 2016;125:585-93.

15. Fotticchia I, Fotticchia T, Mattia CA, Netti PA, Vecchione R, Giancola C. Thermodynamic signature of secondary nano-emulsion formation by isothermal titration calorimetry. Langmuir. 2014;30:14427-33.

16. Pagano B, Fotticchia I, De Tito S, Mattia CA, Mayol L, Novellino E, et al. Selective binding of distamycin a derivative to G-quadruplex structure $[\mathrm{d}(\mathrm{TGGGGT})]_{4}$. J Nucleic Acids. 2010;2010:247137.

17. Ladbury JE. Calorimetry as a tool for understanding biomolecular interactions and an aid to drug design. Biochem Soc Trans. 2010;38:888-93.

18. Hipólito-Nájera AR, del Rosario M-H, Rojas-Hernández A, Gómez-Balderas R. Interaction of indomethacin-cyclodextrins in water by UV-Vis and ITC. J Incl Phenom Macrocycl Chem. 2019;95:55-62.

19. Uria-Canseco E, Perez-Casas S, Navarrete-Vázquez G. Thermodynamic characterization of the inclusion complexes formation between antidiabetic new drugs and cyclodextrins. J Chem Thermodyn. 2019;129:55-60.

20. Navarrete-Vazquez G, Paoli P, León-Rivera I, Villalobos-Molina R, Medina-Franco JL, Ortiz-Andrade R, et al. Synthesis, in vitro and computational studies of protein tyrosine phosphatase 
1B inhibition of a small library of 2-arylsulfonylaminobenzothiazoles with antihyperglycemic activity. Bioorg Med Chem. 2009; 17:3332-41.

21. Amaro B, Alves C, Ferreira G, Carvalho P, da Silva J, Souza C, et al. Multifunctionality of $\beta \mathrm{CD} / \mathrm{Ofloxacin}$ and $\mathrm{HP} \beta \mathrm{CD} / \mathrm{Ofloxacin}$ complexes: improvement of the antimicrobial activity and apoptosis induction on lung adenocarcinoma A549 cells. J Braz Chem Soc. 2020;31:2628-37.

22. Couto VM, de Oliveira-Nascimento L, Cabeça LF, Geraldes DC, Costa JSR, Riske KA, et al. Capsaicin-cyclodextrin complex enhances mepivacaine targeting and improves local anesthesia in inflamed tissues. Int J Mol Sci. 2020;21:1-17.

23. Guimarães PPG, de Menezes AC, Teixeira KIR, Denadai ÂML, Fills RA, Cortés ME, et al. Enhanced efficacy against bacterial biofilms via host:guest cyclodextrin-doxycycline inclusion complexes. J Incl Phenom Macrocycl Chem. 2021. https://doi.org/10. 1007/s10847-020-01041-7.

24. Sachdeva V, Roy A, Bharadvaja N. Current prospects of nutraceuticals: a review. Curr Pharm Biotechnol. 2020;21:884-96.

25. Tian L, Cao J, Zhao T, Liu Y, Khan A, Cheng G. The bioavailability, extraction, biosynthesis and distribution of natural dihydrochalcone: phloridzin. Int J Mol Sci. 2021;22:962.

26. Zhang C-L, Liu J-C, Yang W-B, Chen D-L, Jiao Z-G. Experimental and molecular docking investigations on the inclusion mechanism of the complex of phloridzin and hydroxypropyl- $\beta$ cyclodextrin. Food Chem. 2017;215:124-8.

27. D’Aria F, Serri C, Niccoli M, Mayol L, Quagliariello V, Iaffaioli RV, et al. Host-guest inclusion complex of quercetin and hydroxypropyl- $\beta$-cyclodextrin: a calorimetric study. J Therm Anal Calorim. 2017;130:451-6.

28. Xavier-Júnior FH, Tavares CT, Rabello MM, Hernandes MZ, Bezerra BP, Ayala AP, et al. Elucidation of the mechanism of complexation between oncocalyxone A and cyclodextrins by isothermal titration calorimetry and molecular modeling. J Mol Liq. 2019;274:165-72.

29. da Silva RE, et al. Antimicrobial and antibiofilm activity of the benzoquinone oncocalyxone A. Microb Pathog. 2020;149:104513.

30. Buko V, Zavodnik I, Palecz B, Stepniak A, Kirko S, Shlyahtun A, et al. Betulin/2-hydroxypropyl- $\beta$-cyclodextrin inclusion complex: physicochemical characterization and hepatoprotective activity. J Mol Liq. 2020;309:113-8.

31. Andreadelis I, Chatziathanasiadou MV, Ntountaniotis D, Valsami G, Papaemmanouil C, Christodoulou E, et al. Charting the structural and thermodynamic determinants in phenolic acid natural product-cyclodextrin encapsulations. J Biomol Struct Dyn. 2020;39:2642-58.

32. Wahl J, Furuishi T, Yonemochi E, Meinel L, Holzgrabe U. Characterization of complexes between phenethylamine enantiomers and $\beta$-cyclodextrin derivatives by capillary electrophoresis-determination of binding constants and complex mobilities. Electrophoresis. 2017;38:1188-200.

33. Liu M, Zheng Y, Wang C, Xie J, Wang B, Wang Z, et al. Improved stability of (+)-catechin and (-)-epicatechin by complexing with hydroxypropyl- $\beta$-cyclodextrin: Effect of $\mathrm{pH}$, temperature and configuration. Food Chem. 2016;196:148-54.

34. Maurya PK, Rizvi SI. Protective role of tea catechins on erythrocytes subjected to oxidative stress during human aging. Nat Prod Res. 2009;23:1072-9.

35. Nagarajan $S$, Nagarajan $R$, Braunhut $S$, Bruno F, McIntosh D, Samuelson L, et al. Biocatalytically oligomerized epicatechin with potent and specific anti-proliferative activity for human breast cancer cells. Molecules. 2008;13:2704-16.

36. Usacheva T, Kabirov D, Beregova D, Gamov G, Sharnin V, Biondi M, et al. Thermodynamics of complex formation between hydroxypropyl- $\beta$-cyclodextrin and quercetin in water-ethanol solvents at T $=298.15 \mathrm{~K}$. J Therm Anal Calorim. 2019;138:417-24.

37. Cai C, Liu M, Yan H, Zhao Y, Shi Y, Guo Q, et al. A combined calorimetric, spectroscopic and molecular dynamic simulation study on the inclusion complexation of (E)-piceatannol with hydroxypropyl- $\beta$-cyclodextrin in various alcohol + water cosolvents. J Chem Thermodyn. 2019;132:341-51.

38. Kabirov D, Silvestri T, Niccoli M, Usacheva T, Mayol L, Biondi $\mathrm{M}$, et al. Phase solubility and thermoanalytical studies of the inclusion complex formation between curcumin and hydroxypropyl- $\beta$ cyclodextrin in hydroalcoholic solutions. J Therm Anal Calorim. 2020;1-7.

39. Paul BK, Ghosh N, Mukherjee S. Interaction of bile salts with $\beta$-cyclodextrins reveals nonclassical hydrophobic effect and enthalpy-entropy compensation. J Phys Chem B. 2016;120:3963-8.

40. Schönbeck C, Holm R. Exploring the origins of enthalpy-entropy compensation by calorimetric studies of cyclodextrin complexes. J Phys Chem B. 2019;123:6686-93.

41. Grunwald E, Steel C. Solvent reorganization and thermodynamic enthalpy-entropy compensation. J Am Chem Soc. 1995; 117:5687-92.

42. Breiten B, Lockett MR, Sherman W, Fujita S, Al-Sayah M, Lange $\mathrm{H}$, et al. Water networks contribute to enthalpy/entropy compensation in protein-ligand binding. J Am Chem Soc. 2013;135:15579-84.

43. Dragan AI, Read CM, Crane-Robinson C. Enthalpy-entropy compensation: the role of solvation. Eur Biophys J. 2017;46:301-8.

44. Walker $\mathrm{CH}$. The hydrophobic effect: formation of micelles and biological membranes. FEBS Lett. 1981;124:127-127.

45. Sandilya AA, Natarajan U, Priya MH. Molecular view into the cyclodextrin cavity: structure and hydration. ACS Omega. 2020;5:25655-67.

Publisher's Note Springer Nature remains neutral with regard to jurisdictional claims in published maps and institutional affiliations. 